\title{
RNAi keeps Atf1-bound stress response genes in check at nuclear pores
}

\author{
Katrina J. Woolcock, ${ }^{1,2}$ Rieka Stunnenberg, ${ }^{1,2}$ Dimos Gaidatzis, ${ }^{1,2,3}$ Hans-Rudolf Hotz, ${ }^{1,2,3}$ \\ Stephan Emmerth, ${ }^{1,2}$ Pierre Barraud, ${ }^{4}$ and Marc Bühler ${ }^{1,2,5}$ \\ ${ }^{1}$ Friedrich Miescher Institute for Biomedical Research, 4058 Basel, Switzerland; ${ }^{2}$ University of Basel, 4003 Basel, Switzerland; \\ ${ }^{3}$ Swiss Institute of Bioinformatics, 4058 Basel, Switzerland; ${ }^{4}$ Institute of Molecular Biology and Biophysics, ETH Zürich, \\ CH-8093 Zürich, Switzerland
}

\begin{abstract}
RNAi pathways are prevalent throughout the eukaryotic kingdom and are well known to regulate gene expression on a post-transcriptional level in the cytoplasm. Less is known about possible functions of RNAi in the nucleus. In the fission yeast Schizosaccharomyces pombe, RNAi is crucial to establish and maintain centromeric heterochromatin and functions to repress genome activity by a chromatin silencing mechanism referred to as cotranscriptional gene silencing (CTGS). Mechanistic details and the physiological relevance of CTGS are unknown. Here we show that RNAi components interact with chromatin at nuclear pores to keep stress response genes in check. We demonstrate that RNAi-mediated CTGS represses stress-inducible genes by degrading mRNAs under noninduced conditions. Under chronic heat stress conditions, a Dicer thermoswitch deports Dicer to the cytoplasm, thereby disrupting CTGS and enabling expression of genes implicated in the acquisition of thermotolerance. Taken together, our work highlights a role for nuclear pores and the stress response transcription factor Atf1 in coordinating the interplay between the RNAi machinery and the $S$. pombe genome and uncovers a novel mode of RNAi regulation in response to an environmental cue.
\end{abstract}

[Keywords: RNAi; CTGS; NPC; stress response; thermoswitch; Atf1 transcription factor]

Supplemental material is available for this article.

Received January 7, 2012; revised version accepted February 24, 2012.

The fission yeast Schizosaccharomyces pombe has been an invaluable model to study the assembly of centromeric heterochromatin, which depends on the processing of long noncoding repeat RNAs into double-stranded siRNAs by Dcr1 (Volpe et al. 2002; Moazed 2009). Double-stranded siRNAs are found in the ARC chaperone complex (consisting of Ago1, Arb1, and Arb2), which is involved in siRNA maturation (Buker et al. 2007). Mature, singlestranded siRNAs are found in the RNA-induced transcriptional silencing complex (RITS; consisting of Agol, Chp1, and Tas3) (Verdel et al. 2004) and function to target RITS to nascent chromatin-bound transcripts. Subsequently, this recruits Clr4, the enzyme that methylates histone $\mathrm{H} 3$ at Lys 9 (H3K9). H3K9 methylation, a hallmark of heterochromatin, is crucial to stabilize the association of RITS with chromatin via Chp1, triggering a self-enforcing positive feedback mechanism in which RITS recruits the Rdp1-containing RNA-directed RNA polymerase complex (RDRC) (Motamedi et al. 2004). This activates siRNA amplification, which eventually leads to high levels of H3K9

${ }^{5}$ Corresponding author.

E-mail marc.buehler@fmi.ch.

Article published online ahead of print. Article and publication date are online at http://www.genesdev.org/cgi/doi/10.1101/gad.186866.112. methylation and functional heterochromatin at centromeric repeats. Although this pathway has been investigated in great detail, the mechanisms by which the RNAi machinery is initially targeted to centromeric repeats have remained elusive (Halic and Moazed 2010; Shanker et al. 2010).

Whereas the involvement of RNAi in the assembly of centromeric heterochromatin is well established, additional roles remain ill-defined. Recently, we obtained evidence that the RNAi pathway might also function to repress genomic elements other than the well-studied regions of constitutive heterochromatin. We proposed a cotranscriptional gene silencing (CTGS) model, in which RNAi functions in direct association with euchromatin to trigger RNA decay (Woolcock et al. 2011). The physiological relevance of this mode of genome regulation and, similar to centromeric heterochromatin, how specific targeting of RNAi components to particular regions in the genome is achieved are not known.

\section{Results}

Nuclear RNA turnover proteins physically associate with the S. pombe genome

An inherent difficulty in studying the cross-talk between the RNAi machinery and chromatin has been that some 
RNAi components hardly cross-link to chromatin. A solution to this problem is DNA adenine methyltransferase identification (DamID), a highly sensitive method that allowed us to map Dcrl interactions with the genome (Woolcock et al. 2011). To profile other RNA turnover proteins implicated in chromatin silencing, we produced and compared genome-wide binding maps for Dcr1, Rdp1, Ago1, Arb1, Cid14, Rrp6, and the heterochromatin protein $\mathrm{HP} 1^{\text {Swi6 }}$ by DamID in combination with tiling arrays (Fig. 1A). Of the RNAi proteins, Agol and Rdp1 show a high enrichment at major heterochromatic regions, as observed for $\mathrm{HP} 1^{\text {Swi6 }}$. Dcrl is also enriched at centromeric heterochromatin but not at other heterochromatic loci. Surprisingly, Arb1 is not enriched at any region. Thus, whereas Dcr1, RITS, and RDRC operate in close proximity to heterochromatin, ARC is the only RNAi complex that seems to function further away (Fig. 1A).

The Cid14 nucleotidyltransferase and the RNA exosome have been recently suggested to be involved in siRNA 3' end processing by untemplated nucleotide addition and trimming, respectively (Halic and Moazed 2010). Consistent with an involvement in siRNA maturation, Cid14 shows an enrichment similar to that observed for Dcrl at centromeric heterochromatin (Fig. 1A). This indicates that siRNA maturation by the exosome and Cid14 is initiated in association with chromatin. Interestingly, the nucleusspecific 3'-to-5' exoribonuclease Rrp6 is depleted at centromeres. Therefore, trimming of siRNAs is most likely mediated by another exonuclease, which is consistent with the observation that centromeric siRNA levels are drastically reduced in cid14s but not $\operatorname{rrp} 6 \Delta$ cells (Buhler et al. 2007). In contrast to centromeric heterochromatin, Rrp6 is slightly enriched at the mating type locus and telomeres, which is in line with its role in RNAi-independent repression at these regions (Buhler et al. 2007).

Although not generally enriched in euchromatin, RNAi factors and Cid14 do associate with particular euchromatic loci. Except for Arb1, all RNAi proteins are enriched to similar levels at long terminal repeats (LTRs), consistent with an increase in RNA from LTRs in RNAi mutants (Fig. 1B; Woolcock et al. 2011). Similar profiles can be observed at regions coding for long intergenic noncoding RNAs (lincRNAs), snoRNAs, and snRNAs, as well as at replication origins. wtf and tf2 retrotransposons are exceptional, as they are specifically enriched by Dcr1 and Cid14 only (Fig. 1B).

Taken together, these DamID experiments provide a comprehensive data set for interactions of the S. pombe genome with nuclear RNA turnover proteins implicated in chromatin silencing. Importantly, we found that the core RNAi machinery associates with many noncoding regions of the genome and certain protein-coding genes.

Interactions between chromatin and the RNAi pathway occur at nuclear pores independently of Argonaute

A unifying feature of RNAi pathways is that Argonautecontaining complexes are guided to their respective targets by small RNAs. Coherent with this paradigm, a transcriptome surveillance mechanism in which Agol associates with random, Dcrl-independent RNA degradation products in $S$. pombe has been recently proposed (Halic and Moazed 2010). These small RNAs are referred to as primal RNAs (priRNAs) and may function to target Agol to chromatin independently of Dcr1 or the H3K9 methylation status. Subsequently, RDRC and Dcr1 would be recruited and the siRNA amplification loop started. Indeed, the high enrichment observed for Rdp1 at centromeres is strongly affected in ago $1 \Delta, d c r 1 \Delta$, and $c l r 4 \Delta$ cells (Fig. 2A), implicating H3K9 methylation and a functional RNAi pathway in the establishment of the positive feedback loop. However, although reduced, the association of Rdpl with chromatin is not completely lost in ago1 $\Delta$, $d c r 1 \Delta$, or clr $4 \Delta$ cells (Fig. 2A). Similarly, the enrichment of Dcr1 with centromeres remains unaffected in ago1 $\Delta$,

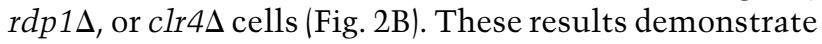
that neither priRNAs nor H3K9 methylation is required for targeting Dcr1 and Rdp1 to centromeric chromatin initially. However, Clr4 stabilizes RITS and RDRC association with heterochromatin, and priRNAs might be required to prime the amplification of centromeric siRNAs.
A
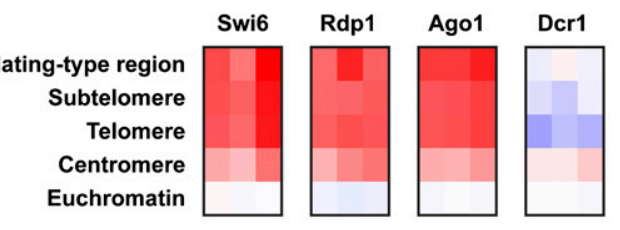

Arb1

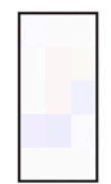

Cid14 Rrp6
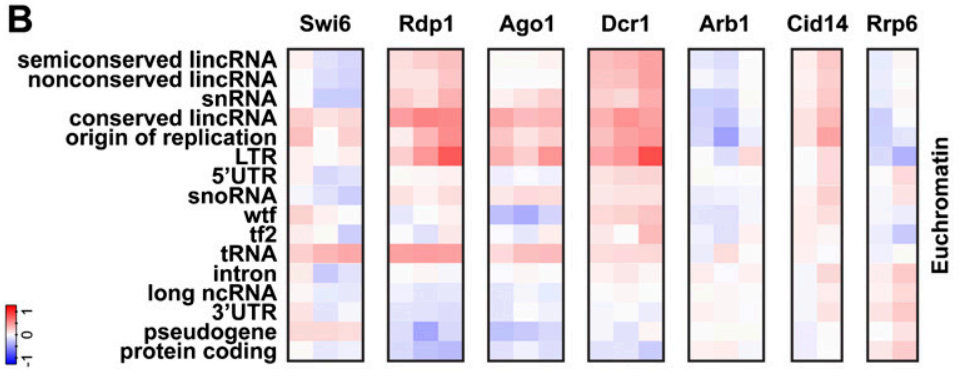

Figure 1. DamID for RNAi and nuclear surveillance components reveals association with genomic regions in both heterochromatin and euchromatin. (A) Enrichments $\left(\log _{2}\right)$ at heterochromatic regions compared with euchromatin. $(B)$ Enrichments $\left(\log _{2}\right)$ at the indicated genomic features present in euchromatin. Individual columns represent biological replicates. (Tf2) Tf2 LTR retrotransposons; (wtf) with Tf2-type LTRs. 
A

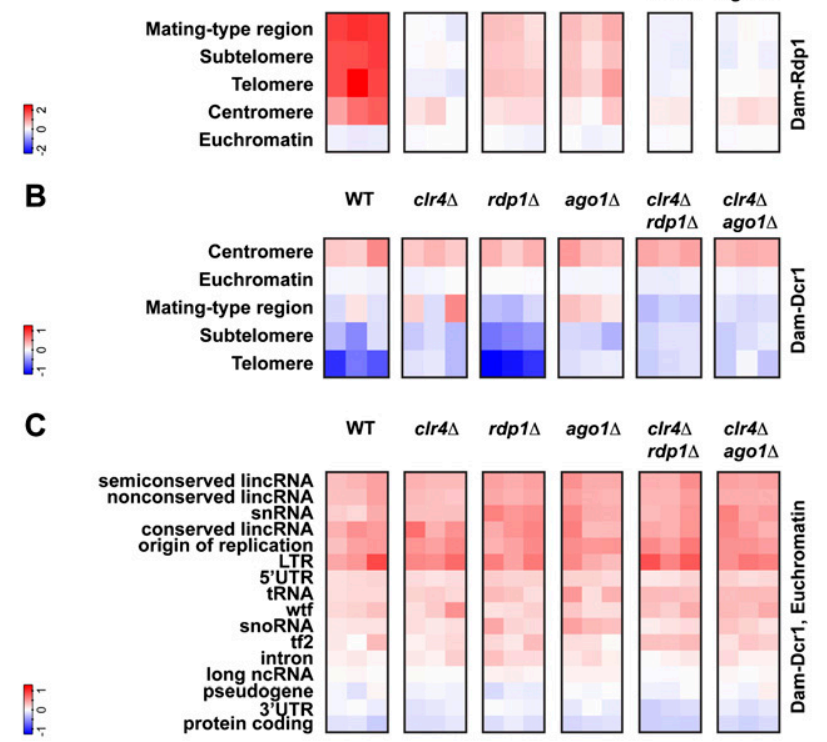

D

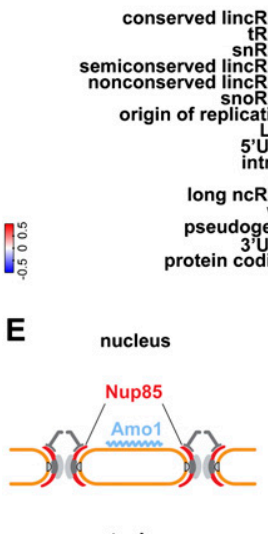

cytoplasm
WT
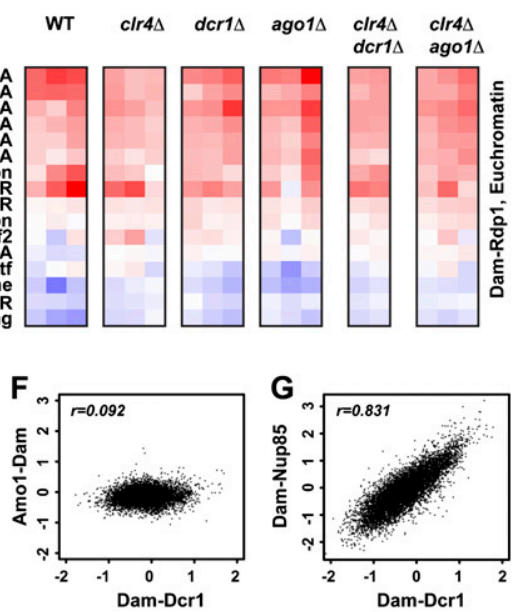

Figure 2. Interactions between chromatin and the RNAi pathway occur at NPCs, independently of small RNAs. $(A-D)$ Rdp1 and Dcr1 enrichments $\left(\log _{2}\right)$ in the mutant backgrounds indicated. Individual columns represent biological replicates. $(E)$ Representation of Nup85 and Amol locations at the nuclear periphery. $(F, G)$ Comparison of Dcrl enrichments $\left(\log _{2}\right)$ at individual features with Amol and Nup85 enrichments, respectively.

A requirement for priRNAs in actively targeting the RNAi machinery to euchromatic sites can also be ruled out because neither Dcr1 nor Rdp1 association with euchromatic loci was affected in ago1s cells (Fig. 2C,D). This raises the question of how RNAi components and specific regions of the genome are brought in close proximity, if not by small RNAs. Intriguingly, Dcrl accumulates at the nuclear periphery in association with pores (Emmerth et al. 2010). Similarly, heterochromatin localizes at the nuclear periphery, prompting us to speculate that the interaction between RNAi components and the $S$. pombe genome might be orchestrated at the nuclear envelope. To test this hypothesis, we performed DamID for two nuclear peripheral proteins (Fig. 2E): Nup85, a scaffold nucleoporin and part of the Nup107-120 complex (Bai et al. 2004), and Amo1, which localizes to the nuclear rim in a punctate pattern that does not overlap with nuclear pore complex (NPC) components (Pardo and Nurse 2005). In contrast to Amol, which does not strongly associate with specific regions of the genome, Nup85 is enriched at several genomic loci (Fig. 2F,G; Supplemental Fig. S1). Intriguingly, comparing the sites associated with Dcrl or Nup85 revealed a strong correlation across the whole genome, demonstrating that NPCs preferentially associate with genomic loci that are also targets of Dcrl (Fig. 2G). Furthermore, Agol seems to be largely dispensable for the association of Nup85 with the genome (Supplemental Fig. S1). These results strongly suggest that the interactions between chromatin and the RNAi pathway are coordinated by one or several components of the NPC and not by small RNAs. Because nuclear pore association of Dcr1 does not depend on a functional RNAi pathway or heterochromatin (Emmerth et al. 2010), Dcrl is likely to interact with NPCs directly.

\section{Dcr1 binds promoter regions and Atf1-bound genes at nuclear pores}

Although genes as a class are not generally enriched in the DamID data (Fig. 1B), we noticed that certain genes are strongly enriched for RNAi components. We observed that Nup85, Dcr1, and Rdp1 are depleted from the intergenic region between convergent genes (Fig. 3A). In contrast, Dcrl and Nup85 are enriched most highly at divergent intergenic regions and intermediately at tandem ones, suggesting that Dcr1 and Nup85 preferentially associate with promoter regions. This is confirmed by the profiles of the average DamID enrichment of Dcrl and Nup85 $1 \mathrm{~kb}$ on either side of the translation start site (Fig. 3B). Rdp1 shows a less-pronounced decrease in enrichment from the $5^{\prime}$ end to the $3^{\prime}$ end.

Intriguingly, we noticed that many of the genes with the highest enrichment for RNAi proteins are involved in responses to stressful conditions. RNAi components show a weak preference for induced core environmental stress response (CESR) genes (Chen et al. 2003) and a strong preference for genes that we refer to as bound by Atf1 under normal conditions (BANC; as judged by chromatin immunoprecipitation [ChIP]-chip data) (Fig. 3C-F; Eshaghi et al. 2010). Atf1 is a basic leucine zipper (bZIP) transcriptional activator and is constitutively bound to its targets (Kon et al. 1997). Upon stress, it becomes phosphorylated and causes an increase in transcription of most of its target genes (Lawrence et al. 2007). Importantly,

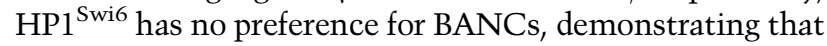
they are euchromatic (Supplemental Fig. S2). Finally, Nup85 shows a strong preference for BANCs, independently of Agol, whereas Amol is depleted (Fig. 3G, $\mathrm{H}$; Supplemental Fig. S2E). These results demonstrate that the RNAi machinery associates with protein-coding genes that are implicated in responses to stressful conditions. These genes are bound by the Atf1 transcription factor and colocalize with the RNAi machinery at NPCs, independently of small RNAs/Argonaute. 
A

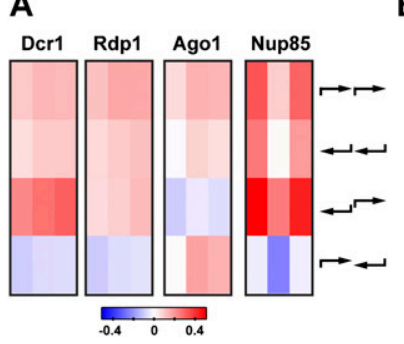

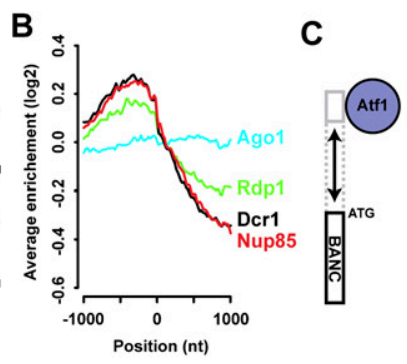
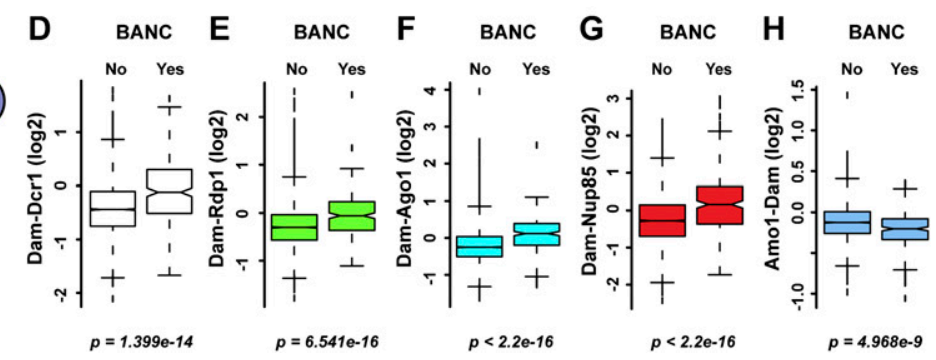

Figure 3. Promoter regions and genes associated with Atf1 colocalize with the RNAi machinery at NPCs. $(A)$ Dcr1, Rdp1, Ago1, and Nup85 enrichments $\left(\log _{2}\right)$ at tandem, divergent, and convergent intergenic regions. Individual columns represent biological replicates. (B) Average enrichments $1 \mathrm{~kb}$ on either side of the beginning of ORFs. One representative replicate is shown for each experiment. (C) BANCs are defined as having an Atf1 ChIP-chip peak under nonstressful conditions in their probable promoter regions (Eshaghi et al. 2010). (D-H) DamID enrichment ( $\left.\log _{2}\right)$ at BANCs ("Yes"; 261 genes) compared with all other genes ("No"; 4715 genes).

\section{RNAi contributes to repression of stress response genes}

The physical association of the core RNAi machinery with stress response genes strongly suggests that RNAi is involved in cellular responses to environmental stress conditions. To test this hypothesis, we did expression profiling in strains lacking $d \mathrm{cr}^{+}$, ago $1^{+}$, or $r d p 1^{+}$. Indeed, BANC genes are significantly up-regulated in all mutants (Fig. 4A-C). Importantly, they are not up-regulated in a swi $6 \Delta$ mutant, confirming that the activation of BANC genes upon deletion of RNAi factors is not an indirect effect of losing heterochromatin, which can be considered a stressful condition (Fig. 4D). Ago1-bound small RNAs that are significantly enriched for BANCs compared with other genes further support the conclusion that the RNAi machinery is directly involved in repressing these loci (Fig. 4E). However, these small RNAs do not seem to be required for the organization of BANCs, Dcr1, and Rdp1 at NPCs, which is independent of Agol, and their functional relevance is unclear. In summary, the RNAi machinery associates with BANC genes independently of small RNAs/Argonaute, although all three core components of the RNAi machinery are required for BANC repression.

To study the effect of stressful insults on expression of Atfl-bound genes in the absence or presence of RNAi, we chose heat shock as a paradigm and monitored expression of candidate heat-shock genes over time. Consistent with impaired repression of BANC genes in the absence of RNAi, heat-shock RNA levels in dcr1s cells, compared with wild type, are higher under noninduced conditions (Fig. 4F; data not shown). Interestingly, RNA polymerase 2 (RNAP2) occupancy changes at some but not all heatshock genes in dcr1 $1 \Delta$ cells (Fig. 4G; Supplemental Fig. S3). Thus, cotranscriptional degradation of RNA might feed back on RNAP2 at some genes. When grown at elevated temperatures, we observed that RNA levels of certain heat-shock genes decrease at a slower rate after their initial induction in $d c r 1 \Delta$ compared with wild-type cells (Fig. $4 \mathrm{H}$,I; data not shown).

These results demonstrate that RNAi, when associated with BANCs, contributes to their repression under normal conditions. This regulation can occur on a truly cotranscriptional RNA decay level, although a regulatory effect on transcription at some genes cannot be ruled out at this point. We propose a model in which Atf1-bound stress response genes are recruited to NPCs and poised for rapid mRNA export, but kept in check by RNAi-mediated CTGS under normal conditions. Upon stress, strongly increased transcription rates simply overcome CTGS, with most transcripts escaping cotranscriptional degradation and accumulating to high levels. After a transient burst of transcription, CTGS contributes to the subsequent decrease in RNA levels.

This model predicts that the association of NPCs with Dcr1 and BANC genes is not disturbed under stressful conditions. Indeed, live-cell imaging of cells in which individual heat-shock genes have been tagged with the LacO/LacI-GFP system did not reveal significant delocalization of heat-shock genes (Fig. 5A,B). Similarly, the association of Dcrl with NPCs was not affected under oxidative stress conditions or short exposure to elevated temperatures (Supplemental Fig. S4). By DamID, associations of Nup85 with the genome remained unchanged at elevated temperatures, as did Dcrl-genome associations under osmotic or oxidative stress conditions (Fig. 5C,D; Supplemental Fig. S5).

\section{Dcr1 translocates to the cytoplasm under chronic heat stress conditions}

In contrast to temporary heat-shock conditions, we observed a striking translocation of Dcrl to the cytoplasm when cells were exposed to chronic heat stress (Fig. 6A). Consistent with this observation, DamID in the DamDcrl strain, but not the Dam-only strain, resulted in much lower DNA concentrations at the end of the protocol when performed at elevated temperatures (Fig. 6B), indicating that Dam-Dcrl has left the nucleus and cannot further methylate DNA. Remarkably, Dcr1 protein levels are comparable at low and high temperatures (Supplemental Fig. S6), and perinuclear Dcrl localization is restored within $1-2 \mathrm{~h}$ when cells are shifted back to $30^{\circ} \mathrm{C}$ after prolonged heat stress, hinting at a possible thermosensitive switch that regulates the nucleo-cytoplasmic distribution of Dcr1 (Fig. 6A).

We previously demonstrated a nucleo-cytoplasmic shuttling function for the dsRNA-binding domain (dsRBD) 
A

$p=2.672 \mathrm{e}-10$
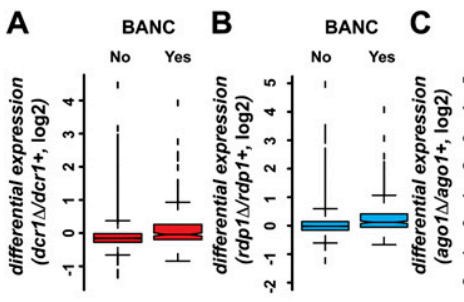

$p=1.356 \mathrm{e}-10$

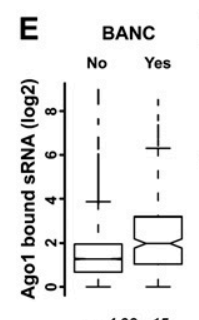

$p=4.98 \mathrm{e}-15$

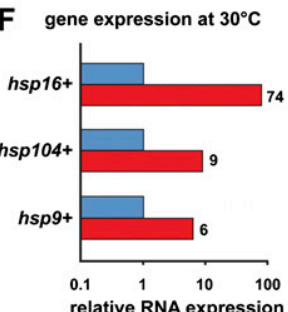

$\square \mathrm{wt} \square \mathrm{dcr} 1 \Delta$

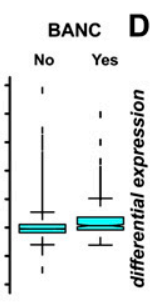

$p=4.17 \mathrm{e}-10$
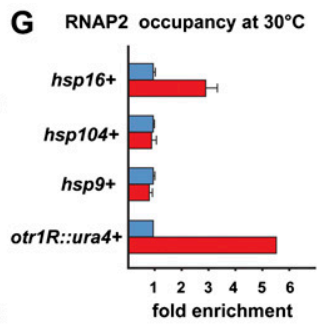

$\square$ wt $\square \mathrm{dcr} 1 \Delta$

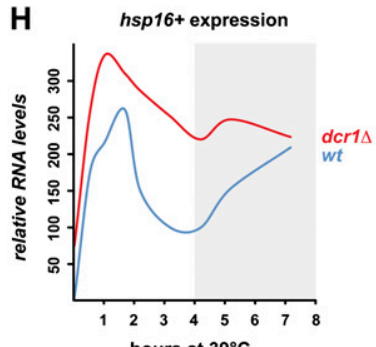

hours at $39^{\circ} \mathrm{C}$

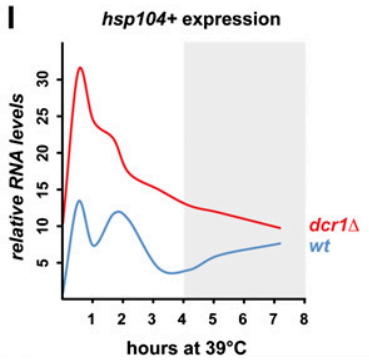

hours at $39^{\circ} \mathrm{C}$

Figure 4. RNAi contributes to cotranscriptional degradation of BANCs. $(A-D)$ Expression analysis by tiling arrays showing differential expression of BANCs ("Yes"; 261 genes) compared with all other genes ("No"; 4715 genes) in $d c r 1 \Delta, r d p 1 \Delta$, ago1s, and swi6s. (E) Small RNA deep-sequencing data (Halic and Moazed 2010) was reannotated to show the number of Ago1bound sRNAs at BANCs compared with all other genes. $(F)$ RNA levels of candidate heat-shock genes in $d c r 1 \Delta$ relative to wild-type cells at $30^{\circ} \mathrm{C}$ were determined by quantitative RTPCR. Actin mRNA was used for normalization. (G) RNAP2 enrichment at candidate heat-shock genes in $d c r 1 \Delta$ relative to wild-type cells at $30^{\circ} \mathrm{C}$. U6 snRNA was used for normalization. otr1R::ura $4^{+}$is located within centromeric heterochromatin and serves as a positive control. $(H, I)$ RNA levels of candidate heatshock genes were measured by quantitative RT-PCR in wildtype and $d c r 1 \Delta$ cells $0 \mathrm{~min}, 30 \mathrm{~min}, 1 \mathrm{~h}, 1 \mathrm{~h} 40 \mathrm{~min}, 2 \mathrm{~h} 10 \mathrm{~min}$, $3 \mathrm{~h} 10 \mathrm{~min}, 4 \mathrm{~h} 10 \mathrm{~min}, 5 \mathrm{~h} 10 \mathrm{~min}$, and $7 \mathrm{~h} 10 \mathrm{~min}$ after shifting to $39^{\circ} \mathrm{C}$. RNA levels were normalized to actin and are represented as fold increase compared with wild-type at $30^{\circ} \mathrm{C}$.

of Dcr1 (Emmerth et al. 2010). However, the physiological relevance of this property has remained mysterious. Intriguingly, the translocation phenotype as a response to chronic heat stress is reminiscent of Dcrl alleles that harbor mutations in the dsRBD. These mutations either abolish the coordination of zinc, which aids proper folding of the dsRBD, or alter the protein-protein interaction surface required for nuclear retention (Fig. 6C; Barraud et al. 2011). Because the integrity of the dsRBD is crucial for nuclear retention of Dcrl, we monitored, by heteronuclear nuclear magnetic resonance (NMR), potential structural changes upon temperature increase. We noticed

a global temperature instability of the domain, revealed by protein precipitation from $\sim 35^{\circ} \mathrm{C}$ and progressive disappearance of NMR signals. Consistent with this observation, differential scanning fluorimetry (Niesen et al. 2007) revealed a melting temperature of $45^{\circ} \mathrm{C}$ for the domain (Fig. 6D). Interestingly, the initiation of the unfolding transition occurs around $34^{\circ} \mathrm{C}-38^{\circ} \mathrm{C}$ - the same temperature at which Dcrl starts leaving the nucleus. Therefore, the Dcrl translocation phenotype that we observed at temperatures $>36^{\circ} \mathrm{C}$ can most likely be attributed to the temperature sensitivity of Dcrl's dsRBD.

We propose that the dsRBD of Dcrl constitutes a thermoswitch that has nuclear retention and NPC interaction properties at temperatures up to $34^{\circ} \mathrm{C}$ (Emmerth et al. 2010; Barraud et al. 2011). At higher temperatures, the protein-protein interaction surface necessary for nuclear retention dissipates and the domain switches to a nuclear export promoter. As a result, RNAi no longer represses BANCs. Consistent with this, BANC genes are significantly up-regulated in Dcrl mutants that cannot fold the dsRBD properly under normal conditions (Fig. $6 \mathrm{E}, \mathrm{F})$. We speculate that this thermoswitch might play an important role in activating chronic heat-shock pathways.

\section{Discussion}

This work points to a critical function for a key stress response transcription factor, Atfl, and nuclear pores in coordinating the interplay between the RNAi machinery and the $S$. pombe genome. That RNAi components colocalize with their targets independently of an Argonaute protein is unusual for RNAi-target interactions. For the establishment of RNAi-mediated CTGS, we foresee the requirement of a scaffold such as the NPC that brings the RNAi machinery and the target locus in close proximity. We propose a model in which transcription factors mediate the specific recruitment of genomic loci such as BANC genes to NPCs, where they meet the RNAi machinery (Fig. 7A). A scaffolding function similar to the one proposed here for NPCs might be attributed to endosomes, multivesicular bodies, or mitochondria in mammalian RNA silencing pathways (Gibbings et al. 2009; Huang et al. 2011; Watanabe et al. 2011).

Besides its crucial role in assembling noncoding repeats into heterochromatin, our work reveals that the $S$. pombe RNAi pathway also contributes to the regulation of protein-coding genes in response to environmental cues. Our results demonstrate that RNAi serves a general function to repress stress response genes. Although we cannot rule out a direct impact on transcription rates at some genes, we favor a CTGS model in which RNAi generally functions on a truly post- or cotranscriptional level by degrading newly synthesized RNA in association with its gene.

Whereas RNAi is involved in the regulation of heatshock gene expression, the RNAi pathway itself is regulated in a unique temperature-dependent manner. Temperatures $>34^{\circ} \mathrm{C}$ trigger a thermoswitch that deports Dcrl to the cytoplasm. Importantly, although reversible, the kinetics of Dcrl translocation to the cytoplasm is slow. Complete loss of perinuclear Dcr1 signal was only observed 
A
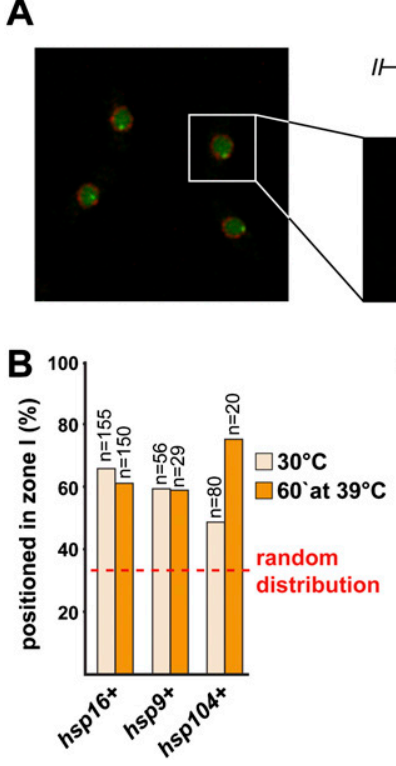

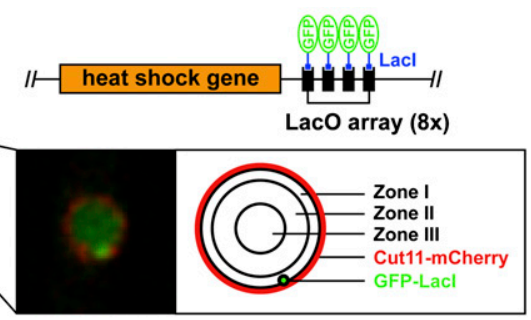

C

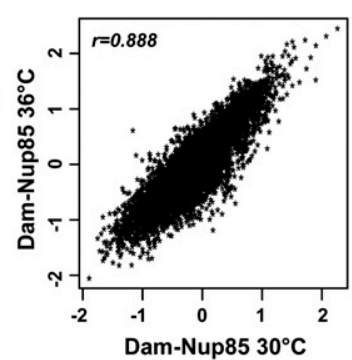

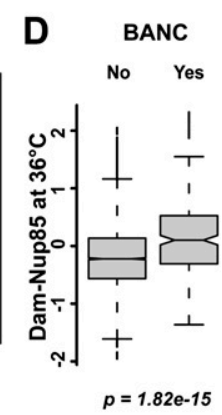

Figure 5. Genes associating with RNAi components do not change their nuclear localization upon temperature increase. (A) Summary of the LacO/LacI-GFP system used to analyze the location of individual heat-shock genes (Taddei et al. 2004). Live cells with GFP-LacI-marked lacO::hsp16 $6^{+}$locus and the nuclear membrane marker mCherry-Cut11 were imaged at $30^{\circ} \mathrm{C}$ (single-plane confocal image). (B) Heat-shock gene localization was assigned to one of three concentric nuclear zones of equal area (shown in $A$ ). Percentage of cells with the GFP focus at the nuclear periphery (zone I) before and after a 1 -h shift to $39^{\circ} \mathrm{C}$ is shown. (Dotted line) Random localization. $(C)$ Comparison of the genome-wide Nup85 enrichment $\left(\log _{2}\right)$ at $30^{\circ} \mathrm{C}$ and $36^{\circ} \mathrm{C}$. $(D)$ BANCs remain highly enriched for Nup 85 at $36^{\circ} \mathrm{C}$. after several hours. This indicates that RNAi-mediated CTGS occurring at nuclear pores is only disrupted completely under chronic heat-shock conditions. Consistent with this idea, two heat-shock genes, hsp $104^{+}$and $h s p 16^{+}$, show reaccumulation of mRNA when incubated for $>4 \mathrm{~h}$ at $39^{\circ} \mathrm{C}$ (Fig. $\left.4 \mathrm{H}, \mathrm{I}\right)$. Intriguingly, these genes have been implicated in the acquisition of thermotolerance (Yoshida and Tani 2005; Senechal et al. 2009), which requires mild pretreatment of cells with sublethal heat stress (Ribeiro et al. 1997). We speculate that transcription of certain genes is activated by mild heat shock, but the response is kept transient by RNAi-mediated CTGS, which remains functional for several hours (Fig. 7B). Upon prolonged incubation at elevated temperatures or recurrent heat shock, the Dcrl thermoswitch could contribute to reactivation of such genes to help the cells tolerate temperatures that would otherwise be deadly (Fig. 7C). Interestingly, loss of silencing at centromeres after chronic heat stress amounts to a maximum increase in RNA of approximately fivefold, much less than the $\sim 100$-fold increase observed in dcr14 cells under normal conditions (data not shown), suggesting the existence of compensatory mechanisms that keep some regions repressed under chronic heat stress despite loss of Dcrl from the nucleus.

Finally, we note that RNAi components have recently been implicated in heat-shock gene regulation in Drosophila melanogaster and that human Dicer has been demonstrated to physically interact with a nuclear pore protein (Ando et al. 2011; Cernilogar et al. 2011). This suggests that mechanisms similar to the ones described here might also be operational in animals.

\section{Material and methods}

Strains and plasmids

Fission yeast strains (grown at $30^{\circ} \mathrm{C}$ in YES medium; MP Biomedicals no. 4101-532) and plasmids used in this study are described in Supplemental Tables S1 and S2. All strains were constructed using a standard PCR-based protocol (Bahler et al. 1998). LacO repeats were inserted into the genome as described previously (Rohner et al. 2008). Constructs on plasmids and in yeast strains were confirmed by sequencing.

\section{DamID}

DamID was done as previously described (Woolcock et al. 2011). For DamID under stress conditions, the cells were grown to $\mathrm{OD}_{600} \sim 0.5$, diluted again to $\mathrm{OD}_{600}=0.08$, and grown again to $\mathrm{OD}_{600} \sim 0.4$. This should dilute out parental methylation so that the new methylation pattern reflects association of the fusion protein with the genome under the stress condition. Heat: $36^{\circ} \mathrm{C}$; osmotic: cells grown in YES with $1 \mathrm{M}$ sorbitol; oxidative: cells grown in YES with $0.5 \mathrm{mM} \mathrm{H}_{2} \mathrm{O}_{2}$.

\section{S. pombe genome annotation}

Two annotations sets ("chromosomal regions" and "genomic elements") were created and can be used in the form of GFF, BED, or Fasta files. The sequence of the genomic DNA is taken from the assembly "pombe_09052011.fasta" (downloaded from ftp://ftp. sanger.ac.uk/pub/yeast/pombe/GFF).

\section{Chromosomal regions}

The three chromosomes were split into centromere, euchromatin, subtelomere, telomere, and mating type regions using the coordinates described in Supplemental Table S4. The centromere ranges were taken from http://www.sanger.ac.uk/Projects/ S_pombe/centromere.shtml. The mating type region and the chromosome ends were divided into regions of high H3K9me2 (telomeres and mating type region) and lower H3K9me2 (subtelomeres) enrichment based on published data (Cam et al. 2005).

For the Fasta files, the "mating_type_region" sequence fragment provided in the genomic DNA file was used instead of the region on chromosome 2 . The euchromatin was extended to include the whole range from 1,644,747 to 4,497,199. The "Spmit" and the "telomeric_contig" sequences from the genomic DNA file were used as well. 


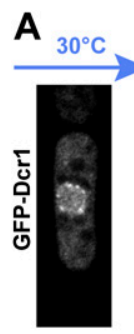

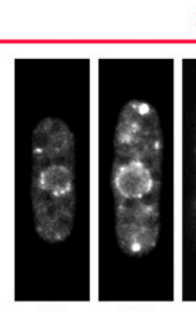

15

30
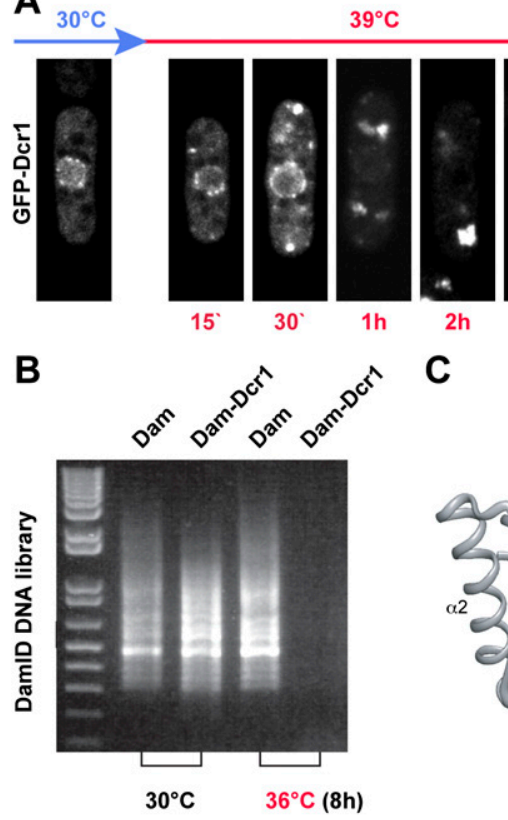

C
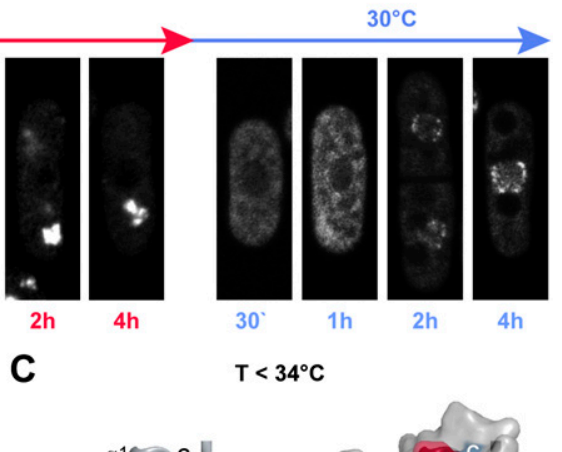

$\mathrm{T}<34^{\circ} \mathrm{C}$
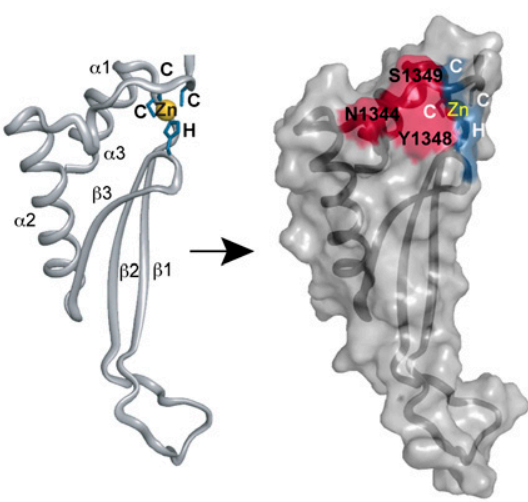

E
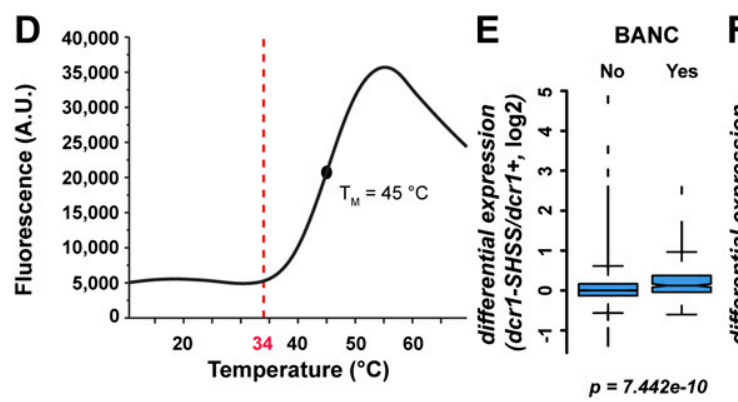

$\mathbf{F}$

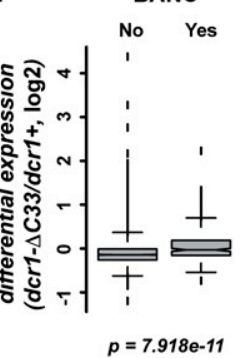

Figure 6. The dsRBD of Dcrl loses nuclear retention properties at elevated temperatures. (A) Fluorescence microscopy of living cells expressing $\mathrm{N}$-terminally tagged GFP-Dcr1. Cells were grown at $30^{\circ} \mathrm{C}$, temperature was shifted to $39^{\circ} \mathrm{C}$, and confocal images were taken at the indicated times (in red). The nuclear peripheral localization of Dcrl is gradually lost and accumulates in bright foci in the cytoplasm, although some nuclear rim signal remains up to $4 \mathrm{~h}$. Recovery of nuclear Dcrl was monitored during $4 \mathrm{~h}$ after shifting the temperature from $39^{\circ} \mathrm{C}$ (cells having been at this temperature overnight) back to $30^{\circ} \mathrm{C}$ (in blue). (B) Much lower DamID library DNA concentrations are obtained for Dam-Dcrl strains when performed at $36^{\circ} \mathrm{C}$ compared with $30^{\circ} \mathrm{C}$ (confirmed by at least four independent replicates). (C) Cartoon representation of the Dcr1 dsRBD is shown on the left (Barraud et al. 2011). Zinccoordinating residues are indicated in blue, and the zinc ion is shown as a yellow sphere. Visualization of the dsRBD fold on the protein surface is shown on the right. The residues highlighted in red form a protein-protein interaction surface that is required to retain Dcrl in the nucleus (Barraud et al. 2011). (D) Thermal unfolding of Dcrl's C-terminal domain shown in $C$ monitored by differential scanning fluorimetry. (Dotted line) Temperature at which unfolding transition initiates. $(E, F)$ Expression analysis by tiling array (Barraud et al. 2011) showing differential expression of BANCs compared with all other genes in a Dcrl mutant in which the dsRBD can no longer coordinate a zinc ion and in a Dcrl mutant lacking the C-terminal 33 amino acids, respectively. Both of these mutants are unable to fold the dsRBD properly and hence lose nuclear retention properties.

\section{Genomic elements}

Initial identification of elements is based on the features described in the GFF file "pombe_09052011.gff" (downloaded from ftp://ftp. sanger.ac.uk/pub/yeast/pombe/GFF).

The sequence ranges of the following features were taken directly from the GFF file. "rep_origin," "LTR," tRNA," "snRNA," and "snoRNA". "rRNA" was built from the rRNA features in the GFF file plus adding SPRRNA.25 (misc_feature). "pre_rRNA" was built from the "misc_feature" features in the GFF file, where the phrase "ribosomal RNA" was present in the attributes column. "pseudogene" was built from the mRNA and "misc_feature" features in the GFF file, where the word "pseudogene" was present in the attributes column, plus adding the mRNA features for SPAC23A1.20 and SPCC622.17. "repeats" was built from the "repeat_region" feature, where the words "wtf" and "tf2" were not present. Lists of " $\mathrm{wtf}$ " and "tf2" identifiers were built from the mRNA features in the GFF file, where the words "wtf" and "tf2," respectively, were present in the attributes column. Sequence regions derived from "pseudogenes," "wtf," and "tf2" elements were removed from the "unspliced_transcripts," "3UTR," and "5UTR" sets built from the mRNA, 3' untranslated region (UTR), and 5' UTR features, respectively. The same restrictions were applied to the "intron" set, in addition to the removal of "tRNA" intron sequences. "tf2" was built by adding the mRNA features from the "tf2" list to the "misc_feature" features in the GFF file, where the phrase "transpos" was present in the attributes column. The "misc_RNA" features were separated based on conservation between different fission yeasts (Rhind et al. 2011; Z Chen, $\mathrm{N}$ Rhind, pers. comm.). "conserved_lincR" were conserved in sequence and location. "semiconserved_lincR" were conserved in location with at least one other species. "nonconserved_lincR" were the remaining "intergenic ncRNA" from the work by Rhind et al. (2011). All remaining "misc_RNA" features (which included all "antisense ncRNA" from the work by Rhind et al. [2011]) were used for "longncRNA."

All resulting GFF files were used to create the BED and Fasta files. In addition, a Fasta file for "mRNA" (i.e., spliced transcripts) was built by combining the sequence for the 5' UTR (if annotated), CDS (one or more per gene), and 3' UTR (if annotated) features for each gene (after removing all features with identifiers present on the "pseudogene," "wtf," and "tf2" lists). The Fasta file for "wtf" was built from sequences of mRNA features from the "wtf" list, where no splicing (i.e., no intron was annotated) occurred. These sequence ranges were also used for the corresponding GFF and BED files. For the remaining identifiers, the sequence was built the same way as the "mRNA" file and was added to the Fasta file. Furthermore, all BED and Fasta files were split into four different files (e.g., "repeats.fa," "repeats_ centromere.fa," "repeats_subtelomere.fa," and "repeats_telomere. $\mathrm{fa}^{\prime \prime}$ ) depending on whether the start of the original feature was within the "euchromatin," "centromere," "subtelomere," and 


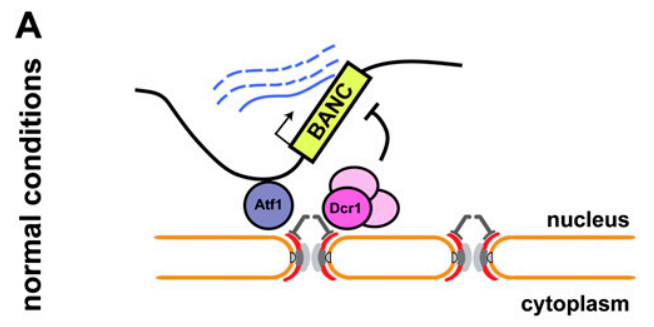

B
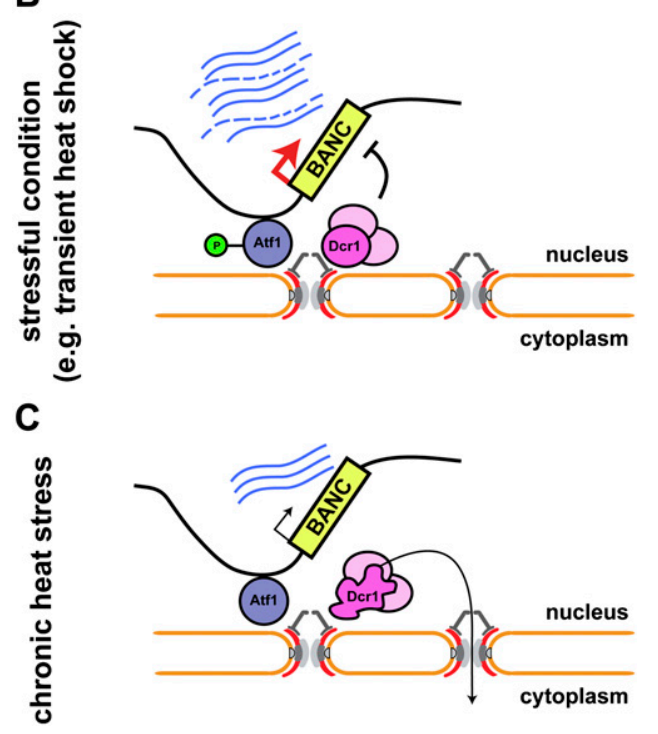

Figure 7. Proposed model for RNAi-mediated CTGS of Atf1bound genes (BANCs) at nuclear pores. (A) The RNAi machinery colocalizes with BANCs at pores, contributing to their repression, presumably by degrading the nascent transcripts. (B) Upon stress, Atfl becomes rapidly and transiently phosphorylated and causes strong transcriptional activation of BANCs. After a transient burst of transcription, CTGS remains active and contributes to the transient nature of the stress response. (C) After several hours at elevated temperatures, Dcrl is lost from the nucleus, presumably due to unfolding of its dsRBD. This abrogates CTGS and causes the reaccumulation of some BANCs, which may be important for thermotolerance.

"telomere," respectively, region describe above. The features present on the "mating_type_region," "Spmit," and "telomeric_ contig" sequences were ignored. The Fasta file for the mitochondrion (i.e., "Spmit") was added as one separate sequence.

\section{DamID analysis}

DamID analysis was done as previously described (Woolcock et al. 2011) but using the above annotation. Intergenic regions were generated as previously described (Woolcock et al. 2011), except that regions interrupted by an LTR were also excluded from the analysis to prevent the high enrichments found at LTRs from influencing the data. Profiles around translation start sites were as follows: For each transcript, we selected all of the uniquely mapping oligos that overlapped within a region of -1000 to 1000 from the start of the ORF. For each base, from the perspective of the translation start site, we calculated the mean enrichment and smoothed the resulting profiles with a lowess normalizer. Profiles from multiple samples were made comparable by subtracting their respective means.

\section{Expression profiling}

RNA isolation and processing were done as previously described (Emmerth et al. 2010). All tiling arrays were processed in $\mathrm{R}$ (Ihaka and Gentleman 1996) using bioconductor (Gentleman et al. 2004) and the packages tilingArray (Huber et al. 2006) and preprocessCore. The arrays were RMA background-corrected, quantile-normalized, and $\log 2$-transformed on the oligo level using the following command: expr $<-\log 2$ \{normalize. quantiles[rma.background.correct/exprs/readCel2eSet[filenames, rotated=TRUE]\}]|\}. Oligo coordinates were intersected with the genome annotation and used to calculate average expression levels for individual genomic features (excluding those with $<10$ oligos) as well as broader annotation categories. In the latter case, multimapping oligos were counted only once per category (avoiding multiple counts from the same oligo).

\section{Statistical analysis}

Box plots and scatter plots were produced in $\mathrm{R}$ and show the average of at least two biological replicates for all experiments unless otherwise stated. All $P$-values were generated using the $\mathrm{R}$ command t.test.

\section{RNA isolation, cDNA synthesis, and quantitative RT-PCR}

RNA isolation, cDNA synthesis, and quantitative RT-PCR were done as previously described (Emmerth et al. 2010). Primer pairs used for PCR reactions can be found in Supplemental Table S3.

\section{ChIP}

PolII ChIP was performed as previously described (Buhler et al. 2006) using the 8GW16 antibody (Covance) and M-280 sheep anti-mouse IgG Dynabeads (Invitrogen). Primer pairs used for PCR reactions can be found in Supplemental Table S3.

\section{Live fluorescence microscopy}

Images were acquired with a LSM710 laser-scanning confocal microscope equipped with a multiline argon 458/488/514-nm (25-mW) laser and a Plan-Apochromat $63 \times / 1,40$ oil DIC M27 objective. Images were processed with ImageJ and Adobe Photoshop. In zoning assays, a second channel was added to visualize cut11-mCherry using the DPSS 561-nm (15-mW) laser. In addition, Z-stacks of 200-nm step size were taken and distance measurements of the foci were extracted using the plug-in "PointPicker" of ImageJ. Assignment of zones and statistical analysis was done as previously described (Taddei et al. 2004). The cells were imaged in a Ludin chamber coated with Lectin (BS-1, Sigma). For heat-shock conditions, the microscope was preheated to $39^{\circ} \mathrm{C}$, cells were kept in a Ludin chamber, and pictures were acquired at different time points.

To monitor Dcrl relocalization in response to temperature changes, cells expressing N-terminally GFP-tagged Dcrl driven by the nmt $1(3 \times)$ promoter were grown in YES at $30^{\circ} \mathrm{C}$ or $39^{\circ} \mathrm{C}$. After the respective temperature shift, aliquots from log phase cells were taken for each time point and spread onto agarose patches containing YES medium with 3\% glucose. Images were acquired using 3.5\% laser power (argon, $488 \mathrm{~nm}$ ) with a gain of 700, a pinhole of $1.5 \mathrm{AU}$, and an averaging of 4 . The microscope was preheated to $30^{\circ} \mathrm{C}$ for the recovery experiments and to $39^{\circ} \mathrm{C}$ in the case of heat induction.

\section{$N M R$ and differential scanning fluorimetry (DSF)}

Protein samples for NMR and DSF were prepared as previously described (Barraud et al. 2011). NMR spectra were recorded with 
a $0.2 \mathrm{mM}{ }^{15} \mathrm{~N}$-labeled sample at temperatures ranging from 298 $\mathrm{K}$ to $318 \mathrm{~K}$ in a buffer containing $25 \mathrm{mM} \mathrm{NaPi}$ (pH 7.0), $75 \mathrm{mM}$ $\mathrm{KCl}, 2 \mathrm{mM}$ DTT, and $10 \mathrm{mM} \mathrm{ZnCl}_{2}$ on Bruker AVIII-500 MHz. Thermal unfolding was monitored in the same buffer conditions by DSF in the presence of SYPRO orange using a real-time PCR instrument (Bio-Rad CFX96). Excitation and emission wave lengths were $492 \mathrm{~nm}$ and $610 \mathrm{~nm}$, respectively. Melting temperature value $\left(T_{M}\right)$ was calculated using the first derivative of the unfolding transition.

\section{Accession codes}

All data sets were deposited under accession number GSE36214 (NCBI Gene Expression Omnibus).

\section{Acknowledgments}

We thank Yukiko Shimada and Nathalie Laschet for technical assistance, Laurent Gelman for assistance with light microscopy, Stéphane Thiry for hybridizing tiling arrays, Tim Roloff for help with quality control and archiving of the DamID data sets, Susan Gasser and Peter Meister for strains and plasmids, and Philip Knuckles for comments on the manuscript. Research in the laboratory of M.B. is supported by the Swiss National Science Foundation, the European Research Council, and the Gebert Rüf Stiftung. The Friedrich Miescher Institute for Biomedical Research is supported by the Novartis Research Foundation.

\section{References}

Ando Y, Tomaru Y, Morinaga A, Burroughs AM, Kawaji $\mathrm{H}$, Kubosaki A, Kimura R, Tagata M, Ino Y, Hirano $H$, et al. 2011. Nuclear pore complex protein mediated nuclear localization of dicer protein in human cells. PLOS ONE 6: e23385. doi: 10.1371/journal.pone.0023385.

Bahler J, Wu JQ, Longtine MS, Shah NG, McKenzie A 3rd, Steever AB, Wach A, Philippsen P, Pringle JR. 1998. Heterologous modules for efficient and versatile PCR-based gene targeting in Schizosaccharomyces pombe. Yeast 14: 943951.

Bai SW, Rouquette J, Umeda M, Faigle W, Loew D, Sazer S, Doye V. 2004. The fission yeast Nup107-120 complex functionally interacts with the small GTPase Ran/Spil and is required for mRNA export, nuclear pore distribution, and proper cell division. Mol Cell Biol 24: 6379-6392.

Barraud P, Emmerth S, Shimada Y, Hotz HR, Allain FH, Buhler M. 2011. An extended dsRBD with a novel zinc-binding motif mediates nuclear retention of fission yeast Dicer. EMBO J 30: 4223-4235.

Buhler M, Verdel A, Moazed D. 2006. Tethering RITS to a nascent transcript initiates RNAi- and heterochromatin-dependent gene silencing. Cell 125: 873-886.

Buhler M, Haas W, Gygi SP, Moazed D. 2007. RNAi-dependent and -independent RNA turnover mechanisms contribute to heterochromatic gene silencing. Cell 129: 707-721.

Buker SM, Iida T, Buhler M, Villen J, Gygi SP, Nakayama J, Moazed D. 2007. Two different Argonaute complexes are required for siRNA generation and heterochromatin assembly in fission yeast. Nat Struct Mol Biol 14: 200-207.

Cam HP, Sugiyama T, Chen ES, Chen X, FitzGerald PC, Grewal SI. 2005. Comprehensive analysis of heterochromatin- and RNAi-mediated epigenetic control of the fission yeast genome. Nat Genet 37: 809-819.

Cernilogar FM, Onorati MC, Kothe GO, Burroughs AM, Parsi KM, Breiling A, Sardo FL, Saxena A, Miyoshi K, Siomi H, et al. 2011. Chromatin-associated RNA interference components contribute to transcriptional regulation in Drosophila. Nature 480: 391-395.

Chen D, Toone WM, Mata J, Lyne R, Burns G, Kivinen K, Brazma A, Jones N, Bahler J. 2003. Global transcriptional responses of fission yeast to environmental stress. Mol Biol Cell 14: 214-229.

Emmerth S, Schober H, Gaidatzis D, Roloff T, Jacobeit K, Buhler M. 2010. Nuclear retention of fission yeast dicer is a prerequisite for RNAi-mediated heterochromatin assembly. Dev Cell 18: 102-113.

Eshaghi M, Lee JH, Zhu L, Poon SY, Li J, Cho KH, Chu Z, Karuturi RK, Liu J. 2010. Genomic binding profiling of the fission yeast stress-activated MAPK Sty1 and the bZIP transcriptional activator Atf1 in response to $\mathrm{H}_{2} \mathrm{O}_{2}$. PLOS ONE 5: e11620. doi: 10.1371/journal.pone.0011620.

Gentleman RC, Carey VJ, Bates DM, Bolstad B, Dettling M, Dudoit S, Ellis B, Gautier L, Ge Y, Gentry J, et al. 2004. Bioconductor: Open software development for computational biology and bioinformatics. Genome Biol 5: R80. doi: 10.1186/ gb-2004-5-10-r-80.

Gibbings DI, Ciaudo C, Erhardt M, Voinnet O. 2009. Multivesicular bodies associate with components of miRNA effector complexes and modulate miRNA activity. Nat Cell Biol 11: 1143-1149.

Halic M, Moazed D. 2010. Dicer-independent primal RNAs trigger RNAi and heterochromatin formation. Cell 140: 504-516.

Huang H, Gao Q, Peng X, Choi SY, Sarma K, Ren H, Morris AJ, Frohman MA. 2011. piRNA-associated germline nuage formation and spermatogenesis require MitoPLD profusogenic mitochondrial-surface lipid signaling. Dev Cell 20: 376-387.

Huber W, Toedling J, Steinmetz LM. 2006. Transcript mapping with high-density oligonucleotide tiling arrays. Bioinformatics 22: $1963-1970$.

Ihaka R, Gentleman R. 1996. R: A language for data analysis and graphics. J Comput Graph Statist 5: 299-314.

Kon N, Krawchuk MD, Warren BG, Smith GR, Wahls WP. 1997. Transcription factor Mts1/Mts2 (Atf1/Pcr1, Gad7/ Pcr1) activates the M26 meiotic recombination hotspot in Schizosaccharomyces pombe. Proc Natl Acad Sci 94: 13765-13770.

Lawrence CL, Maekawa H, Worthington JL, Reiter W, Wilkinson CR, Jones N. 2007. Regulation of Schizosaccharomyces pombe Atf1 protein levels by Sty1-mediated phosphorylation and heterodimerization with Pcr1. J Biol Chem 282: 51605170 .

Moazed D. 2009. Small RNAs in transcriptional gene silencing and genome defence. Nature 457: 413-420.

Motamedi MR, Verdel A, Colmenares SU, Gerber SA, Gygi SP, Moazed D. 2004. Two RNAi complexes, RITS and RDRC, physically interact and localize to noncoding centromeric RNAs. Cell 119: 789-802.

Niesen FH, Berglund H, Vedadi M. 2007. The use of differential scanning fluorimetry to detect ligand interactions that promote protein stability. Nat Protoc 2: 2212-2221.

Pardo M, Nurse P. 2005. The nuclear rim protein Amol is required for proper microtubule cytoskeleton organisation in fission yeast. I Cell Sci 118: 1705-1714.

Rhind N, Chen Z, Yassour M, Thompson DA, Haas BJ, Habib N, Wapinski I, Roy S, Lin MF, Heiman DI, et al. 2011. Comparative functional genomics of the fission yeasts. Science 332: 930-936.

Ribeiro MJ, Reinders A, Boller T, Wiemken A, De Virgilio C. 1997. Trehalose synthesis is important for the acquisition of thermotolerance in Schizosaccharomyces pombe. Mol Microbiol 25: 571-581. 
Rohner S, Gasser SM, Meister P. 2008. Modules for cloning-free chromatin tagging in Saccharomyces cerevisae. Yeast 25: 235-239.

Senechal P, Arseneault G, Leroux A, Lindquist S, Rokeach LA. 2009. The Schizosaccharomyces pombe Hsp104 disaggregase is unable to propagate the [PSI] prion. PLOS ONE 4: e6939. doi: 10.1371/journal.pone.0006939.

Shanker S, Job G, George OL, Creamer KM, Shaban A, Partridge JF. 2010. Continuous requirement for the Clr4 complex but not RNAi for centromeric heterochromatin assembly in fission yeast harboring a disrupted RITS complex. PLoS Genet 6: e1001174. doi: 10.1371/journal.pgen.1001174.

Taddei A, Hediger F, Neumann FR, Bauer C, Gasser SM. 2004 Separation of silencing from perinuclear anchoring functions in yeast Ku80, Sir4 and Esc1 proteins. EMBO J 23: 1301-1312.

Verdel A, Jia S, Gerber S, Sugiyama T, Gygi S, Grewal SI, Moazed D. 2004. RNAi-mediated targeting of heterochromatin by the RITS complex. Science 303: 672-676.

Volpe TA, Kidner C, Hall IM, Teng G, Grewal SI, Martienssen RA. 2002. Regulation of heterochromatic silencing and histone H3 lysine-9 methylation by RNAi. Science 297: 18331837.

Watanabe T, Chuma S, Yamamoto Y, Kuramochi-Miyagawa S, Totoki Y, Toyoda A, Hoki Y, Fujiyama A, Shibata T, Sado T, et al. 2011. MITOPLD is a mitochondrial protein essential for nuage formation and piRNA biogenesis in the mouse germline. Dev Cell 20: 364-375.

Woolcock KJ, Gaidatzis D, Punga T, Buhler M. 2011. Dicer associates with chromatin to repress genome activity in Schizosaccharomyces pombe. Nat Struct Mol Biol 18: 94-99.

Yoshida J, Tani T. 2005. Hsp16p is required for thermotolerance in nuclear mRNA export in fission yeast Schizosaccharomyces pombe. Cell Struct Funct 29: 125-138. 


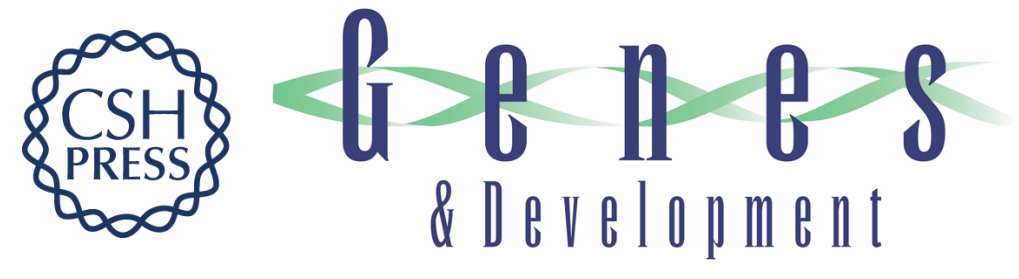

\section{RNAi keeps Atf1-bound stress response genes in check at nuclear pores}

Katrina J. Woolcock, Rieka Stunnenberg, Dimos Gaidatzis, et al.

Genes Dev. 2012, 26: originally published online March 19, 2012

Access the most recent version at doi:10.1101/gad.186866.112

\section{Supplemental http://genesdev.cshlp.org/content/suppl/2012/03/12/gad.186866.112.DC1 \\ Material}

Related Content RNAi in fission yeast finds new targets and new ways of targeting at the nuclear periphery

Daniel Holoch and Danesh Moazed

Genes Dev. April , 2012 26: 741-745

References This article cites 35 articles, 9 of which can be accessed free at: http://genesdev.cshlp.org/content/26/7/683.full.html\#ref-list-1

Articles cited in:

http://genesdev.cshlp.org/content/26/7/683.full.html\#related-urls

\section{License}

Email Alerting

Service

Receive free email alerts when new articles cite this article - sign up in the box at the top right corner of the article or click here.

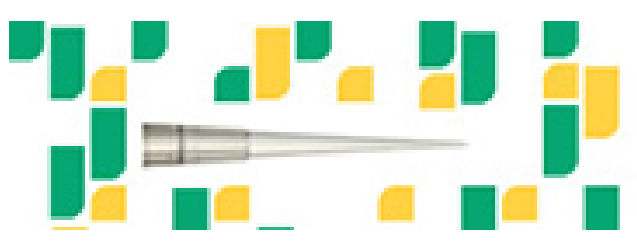

Focused on your science. 